\title{
Deixis, Binding and Presupposition
}

\author{
Nicholas Asher
}

\section{Introduction}

Dynamic semantic accounts of presupposition have proven to quite successful improvements over earlier theories. One great advance has been to link presupposition and anaphora together (van der Sandt 92, Geurts 95), an approach that extends to integrate bridging and other discourse phenomena (Asher and Lascarides 1998a,b). In this extended anaphoric account, presuppositions attach, like assertions, to the discourse context via certain rhetorical relations. These discourse attachments constrain accommodation and help avoid some infelicitous predictions of standard accounts of presupposition. Further, they have interesting and complex interactions with underspecified conditions that are an important feature of the contributions of most presupposition triggers.

Deictic uses of definites, on the other hand, seem at first glance to fall outside the purview of an anaphoric theory of presupposition. There seems to be little that a discourse based theory would have to say. I will argue, however, that a discourse based account can capture how these definites function in conversation. In particular such accounts can clarify the interaction between the uses of such deictic definites and various conversational moves. At least some deictic uses of definites generate presuppositions that are bound to the context via a rhetorical function that I'll call anchoring, which if successful entails a type of knowing how. If this anchoring function is accepted, then the acceptors know how to locate the referent of the definite in the pres??ent context. I'll concentrate here just on definites that refer to spatial locations, where the intuitions about anchoring are quite clear. But I think that this view extends to other deictic uses of definites and has ramifications for an analysis of de re attitudes as well.

\section{Different ways to bind presuppositions}

To set the stage for an analysis of anchoring uses of definites and the role that their presuppositions play there, it is useful to see how varied a role presuppositions of definites play in anaphoric uses of definites. According to "Dynamic" accounts like Heim's familiarity theory (1982), definites presuppose familiar discourse referents. Such presuppositions must be satisfied in the discourse context in the Tarskian sense or must be accommodated (i.e., added) to the discourse context. Van der Sandt (1993) tells us to find these discourse referents via anaphora resolution - i.e., try to bind, and failing that, accommodate. Geurts extends this view by including propositional identity as a means of binding.

Nevertheless, there is much more that can be said about binding. Consider bridging examples like the following: 
(1) a. I met two interesting people last night at a party.

b. The woman was a member of Clinton's Cabinet.

(2) a. John took engine E1 from Avon to Dansville.

b. Then he picked up the boxcar

c. and took it to Broxburn.

Now what happens to the presuppositions generated by the boxcar in (2b)? On standard accounts, we can't bind the boxcar to any discourse entity in the context nor can we satisfy the presupposition that there is a boxcar in the discourse context created by the first sentence. So all the standard theories of presupposition would say that we should accommodate a boxcar in the context. This misses an important aspect of the meaning in this discourse, which we can focus on by asking ourselves: Where ist the boxcar? The discourse based account in Asher and Lascarides (1998a) gets this essential part of the interpretation of (2) by assuming that the presuppositional material introduced by the definite contains some underspecified elements, while the bridging relation is set to identity if this produces a well-defined result (thus incorporating the insights by van der Sandt that binding is preferred), but in this case there is no nonabsurd identification of the boxcar with some other discourse entity to be had. However, in (2b), there is a discourse particle or adverbial then that determines the discourse relation between ( $2 a)$ and $(2 b)$ to be one of Narration. The presence of such a discourse relation between ( $2 a)$ and $(2 b)$ entails that the event described in (2b) is understood as coming after the event described in (2a) and as spatially located in the location in which the event in (2a) terminates (Dansville). The lexical semantics of pick up adds the information that in effect the boxcar is in Dansville. This suffices to determine the bridging relation in this case to be 'in'. Thus, the boxcar is linked to Dansville and that is enough to get the right interpretation. Details of the analysis can be found in Asher and Lascarides (1998a).

With (1), we also see a need to supplement both the Heim and van der SandtGeurts approaches to presupposition. Again we can't bind the woman to any discourse entity in the context nor can we satisfy the presupposition that there is a woman in the discourse context created by the first sentence. So all the standard theories of presupposition would say that we should accommodate a woman in the context. But this again misses an essential component of the interpretation of (1): the woman is one of the two people that I met last night. The discourse based anaphoric account gets this essential part of the interpretation of (1) by a simultaneous resolution of the underspecified bridging and a computation of the discourse connections between the presupposition generated by the woman, the asserted component of (1b) and (1a) accounts for this anaphoric connection and the coherence of the text. More specifically, in this case again, specifying the bridging relation to identity yields an absurdity. But if we specify the bridging relation to be "an element of", we get a coherent discourse and a discourse relation of elaboration between (1a) and (1b). Alternatively, specifying the discourse relation to be Elaboration will coerce the underspecified bridging relation to the appropriate value. ${ }^{1}$

Other examples of complex presupposition binding occur when the presupposition trigger, the expression that generates the presupposition, is itself a discourse particle. That is the case with (3):

I See Asher and Lascarides (1998a) for more details, and also section 4 below. 
John lives in New York too.

Kripke in an unpublished paper observes that (3) can't be uttered in a null context, even if many people are known by the speaker and the audience to live in NY. But accounts like van der Sandt's and Heim's don't predict this. On the other hand, the discourse based account can, of we assume that the presupposition of too is that it generates a specific rhetorical function connecting the assserted content of (3) to some element of the contextually given discourse structure. In the null context there is no element of discourse structure to connect to, and so the presupposition of too can't be fulfilled.

A final example of binding with rhetorical relations reveals that not only are the Heim- van der Sandt - Geurts accounts of presupposition incomplete but they derive wrong interpretations. Consider (4).

(4) a. If a farmer goes to the market, he buys a donkey.

b. Yesterday, Farmer John went to the market.

c. The donkey he bought was expensive.

d. This time the donkey was expensive

e. This time (?)it donkey $_{\text {was }}$ expensive.

Van der Sandt and Geurts must accommodate the existence of a donkey in order to interpret the presupposition of $(4 \mathrm{c}, \mathrm{d}, \mathrm{e})$. But the donkey in (4) depends on an anaphoric link between bought and went. Accommodation yields incorrect results. Heim's (1983) theory yields only the satisfaction of an existential presupposition, not an anaphoric one. We need an appropriate instantiation of the conditional (like (4a'))

(4) a' If John is a farmer and went to market, John bought a donkey

which, when coupled with (4b), gives the donkey referred to in $(4 \mathrm{c})$.

If a farmer goes to Paris, he buys a donkey

Pedro went to Paris. His donkey was expensive.

(6) A Farmer buys a donkey whenever he visits the market.

Farmer John visits the market on Wednesdays. His donkeys are merry.

Similarly, for (4) adding an argument for going-to-the-market events. We might call this inferential binding. The inferential binding in (4abc) falls squarely within the analysis given to the bridging examples and to our anaphoric theory of presuppositions. The rhetorical relation that binds the presupposition to the discourse context is the relation of Defeasible Consequence (Asher and Lascarides 1998b), a natural generalization of Geurts's notion of propositional binding. Defeasible Consequence holds of two propositions $p, q$ iff $q$ is a defeasible consequence of $p{ }^{2}$ This relation will bind the presupposition to both (4a) and (4b) as both are necessary to derive the presupposition that Pedro owns a donkey.

2 Defeasible consequence is defined precisely via a nonmonotonic logic. See for instance Lascarides and Asher 1993, or Asher and Morreau 1991. 
In these examples, however, we cannot specify the bridging relation to identity. So the semantics of the rhetorical relation used to bind the presupposition must determine the bridging relation. In these examples the bridging relation is set to a witnessing relation $\mathrm{W}$. While one term of $\mathrm{W}$ should be the discourse referent introduced by the definite, it is less clear what the other term should be. If we allow reference to quantifiers themselves or their logical forms (it is after all one type of abstract object), then we can take the other term of $\mathrm{W}$ to be the quantifier of which the definite produces an instance. In (4), the appropriate quantifier is a donkey in (4a).

\section{3. “Deictic binding" and Discourse Function}

Not all uses of definite descriptions fit so neatly into an anaphoric theory of presupposition. Definite descriptions have deictic uses within ordinary conversation. Let's take some simple examples:

(7) a. Now pour the mixture into the pan and gently simmer for 10 mins.

b. Move the window to the lower left (on a computer screen).

c. Close the window in the bedroom.

d. You've just checked into a hotel and the clerk says: Your room is up the stairs and right at the end of the corridor.

Many of these definites occur in contexts where, e.g., a window on the computer screen has already been introduced in a previous discourse turn. So the presupposition would be satisfied here by linking the discourse referents introduced by the two NPs. On the other hand, this mere anaphoric connection isn't sufficient to carry out the conversational purpose behind these instructions. In order, for example, to carry out the instruction in $(7 \mathrm{~d})$, the addressee need to be able to find the referent of the description. Similarly for $(7 \mathrm{a}, \mathrm{c})$. The discourse referents introduced by the definites have to be linked or anchored to particular nonlinguistic elements in the visual nonlinguistic context. The case in (7d) is a bit different, but in a way it's more interesting. The definites your room, the corridor have a standard anaphoric analysis but the stairs is somewhat different. We could simply accommodate that there is a corridor on an anaphoric account, but we would miss the intended interpretation - viz. that the stairs be linked to some object in the environment that accomplishes the manifest goal of the speaker, which is that the addressee knows how to get to his room. As one would expect, a standard, dynamic account of presupposition, which treats the presupposition of the definite in (7d) simply by adding it to the context, misses the rhetorical point of the speaker.

This rhetorical function of the presupposition for the speaker in a given context is part of what determines conversation. Consider what happens when this rhetorical function isn't shared by the interpreter or addressee. If the addressee cannot locate the stairs, for instance, it is quite appropriate for him to say:

$$
\text { d'. Where are the stairs? }
$$

We saw earlier that the presupposition of a definite description when resolved can help determine a rhetorical function for the asserted content of a sentence (viz. (lb)), and it seems as though the presupposed material here too has an imprtant role to play in this rhetorical function. But what exactly does it do? 
It's helpful to consider the behavior of such deictic uses of definites within longer stretches of dialogue. Consider the uses of definite descriptions referring to locations or spatially localized entities in the following two dialogues.
a. I: Philippe? (i) C'est Isabelle (ii). Bon, je crois que je me suis un peu per- due (iii).
b. P: Ah bon? Et tu m'appelles d'où?
c. I: Bon, j'en sais rien! Je ne vois pas le nom de la rue.
d. P: Mais tu as fait comment pour y arriver?
e. I: Non, en fait, je suis tout an bout de l'avenue Jean Jaurès, après la grande place (i). C'est une grande rue et il y a un panneau qui indique "Aubervilliers" tout droit (ii). Je ne suis pas sur le bon chemin, non? (iii)
f. P: Attends, je ne vois pas trop où tu es (i). Tu as pris sous la voie ferrée, comme je t'avais dit? (ii)
g. I: D'ici on voit une voie ferrée, an dessus de la place.
h. $\quad$ P: An dessus de la place? Non, ça peut pas être ça!
i. I: Ecoute, l'avenue où tu habitais, c'était bien Jean Jaurès? Et bien, la grande place est juste après, avec le pont suspendu.
j. $\quad$ P: Non, mais ça, ça doit être le périph et pas la voie ferrée!
k. I: Bon, peut-être, d'ici ça se voit pas.
1. P: Tu es à côté de la cité de la musique?
m. I: J'en sais rien, je connais pas la cité de la musique (i). Mais attends, est- ce qu'il fallait que je prenne un deuxième tunnel après celui de chez toi ?
(ii)
n. P: Bien sûr, c'est là que tu passes sous la voie ferrée.

In this dialogue, Isabelle is trying to find her way to Phillippe's new apartment in Paris. There are many definites referring to locations or landmarks; many cannot be bound anaphorically to previous items mentioned in the discourse. The turn (81$\mathrm{m}$ ) is quite interesting. La cité de la musique is a novel definite, with which Isabelle is obviously not familiar, since she says, that she doesn't know the Cité de la Musique. According to standard anaphoric theories of presupposition, one would at his point accommodate the presupposition of the definite, and the discourse would proceed smoothly. But that's not what happens; the discourse doesn't proceed smoothly. Isabelle's response feels as though it entails a presupposition failure - that is, it indicates that the presupposition cannot be satisfactorily treated. With such presupposition failure, there is no possibility of carrying out commands, answering questions, or the like. It has to me the same flavor as orthodox presupposition failures in questions:

What does the present King of France do on Bastille Day?

Just as Isabelle says exasperatedly, "I don't know the Cité de la Musique" ( $8 \mathrm{~m})$, one could respond to this question with a similar exasperated correction: there is no present king of France! Were accommodation possible here, Isabelle would still not be able to answer the question.

If the accommodation of novel uses of definite descriptions is not always an option, how do we understand the "binding" of these novel definites? In the dialogue, there 
are uses of definites that could be bound via identity to a previously mentioned occurrence as in:

(8f,ii) P: $\quad$ Tu as pris sous la voie ferrée comme je t'avais dit?

But interestingly Isabelle does not use this binding alone. In order to be able to answer the question, she must be able to identify the railroad tracks in her immediate environment or as something she passed on her journey. She has to "anchor" the definite to some object in the (nonlinguistic) context. To that end, she offers up an object in her perceptual context with which to bind la voie ferrée.

(8g) I: $\quad$ D'ici on voit une voie ferrée, an dessus de la place.

Interestingly again, Phillippe rejects this contextual anchoring of the railroad tracks in $(8 \mathrm{~h})$; he identifies what she sees as the Boulevard Peripherique. So it looks like deictically used definites do generate familiarity presuppositions; further the satisfaction of the presupposition is accomplished by linking the definite to some object in the nonlinguistic context. Finally, it appears that an upshot of this linking is a mutual belief that both participants in the dialogue are referring to the same object with the description. When this doesn't happen, we get a Correction or some sort of question by the other participants, as Phillippe does in $(8 \mathrm{~h})$.

Exactly what is the nature of this Anchoring ? It's easy enough to see that it involves some sort of de re attitude toward the object, but just saying this doesn't illuminate an important link between Anchoring of a definite in an utterance and the conversational goals of the utterance or of utterances linked to it. Consider

$(8 \mathrm{e}, \mathrm{i})$ I: Non, en fait, je suis tout an bout de l'avenue Jean Jaurès, après la grande place.

au bout de l'avenue Jean Jaurès is a definite with a novel use. In another context accommodation might suffice, but not here with the particular conversational goals of finding out where Isabelle is. Or perhaps, the location denoted by the end of the avenue Jean Jaurès could be bound to some doxastically accessible discourse referent, since Phillippe lived in that neighborhood. But in Phillippe's response to Isabelle (8f.i), he makes it clear that this binding isn't sufficient for him to attain his speech act related goal of knowing where Isabelle is. In order to satisfy his conversational goals he needs a contextual anchoring that will support a de re knowledge claim. What would suffice is a binding of the presupposed location to some location that he is familiar with and can locate on his "cognitive map". And in fact this is what Isabelle wants to do too in view of the goal they both have of getting Isabelle unlost.

That Anchoring must be done in order to achieve the conversational goals is also well-attested in the map corpus dialogues (Edinburgh University). I give a sample here.

(10) a. A: Start at the extinct volcano, and go down round the tribal settlement. And then

b. B: Whereabouts is the tribal settlement?

c. A: It's at the bottom. It's to the left of the extinct volcano.

d. B: Right. How far? 


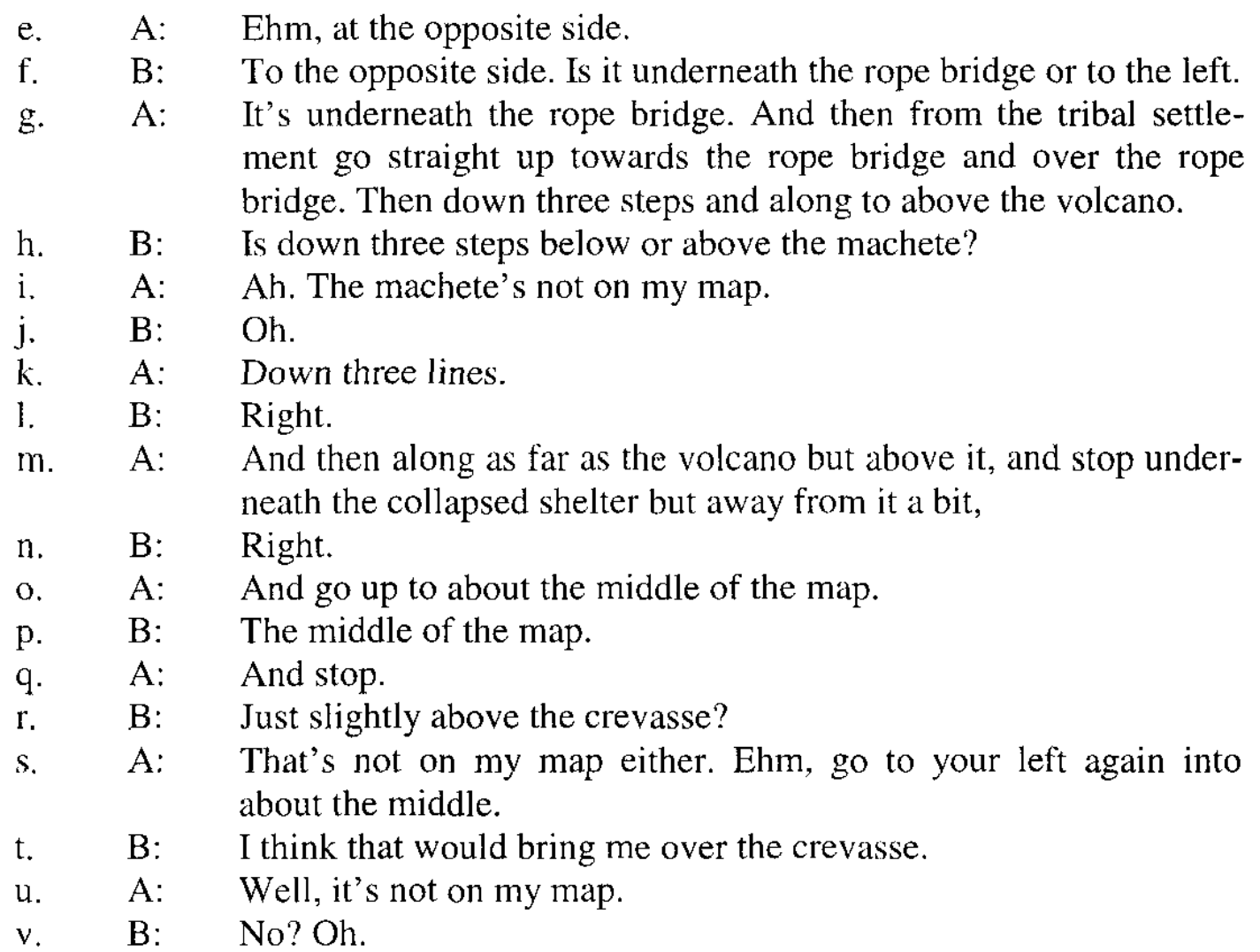

In the MAP Task Corpus dialogues, agent $A$ is trying to get $B$ to a given goal in a game, in which both $A$ and $B$ have a partially accurate map of the terrain to be traversed. In this dialogue the instructions to move to a particular place can only be carried out once either that place or obstacles to be circumvented have been anchored in an appropriate way to their maps. When this Anchoring doesn't take place, then the agents can't give or carry out directions and they have to settle on another means for conveying directions. The directions can only be carried out once the locations involved are appropriately anchored.

I want to draw several conclusions from the discussion of these examples. First, like other uses of definites, deictically used definites do generate familiarity presuppositions. Second these presuppositions are not accommodated or bound in the way familiar from standard, anaphoric theories of presupposition. But that doesn't mean that we have to throw out the machinery of the anaphoric theory. In fact, the discourse based anaphoric theory of presuppositions is very useful: we can understand contextual anchoring as a special sort of presupposition binding; in fact contextual anchoring is a rhetorical function of the presupposed information in these deictic cases. For the discourse to be felicitous, the presupposition generated by a deictically used definite must be tied to some object in the nonlinguistic context such that the interpreter believes he knows how to identify it or make use of that object for some conversationally salient, discourse purpose. The upshot of such anchoring is a mutual belief between speaker and hearer that they are referring to the same object with the description. When this doesn't happen, we get a Correction or some sort of question by the other participants, as Phillippe does in $(8 \mathrm{~h})$, and as Isabelle does in $(8 \mathrm{~m})$.

Phillippes goal is to get de re knowledge, to know where Isabelle is. But this goal itself is subservient to another goal namely that of getting Isabelle unlost. Let's assume for now that Phillippe has acquired this goal. and it is this higher goal that 
tells us what sort of de re knowledge this really is; Phillippe needs to know where Isabelle is so that he can give her directions to get her to her destination. And to give these directions he has to construct a path from Isabelle's present location to her destination and to do that he has to be able to fix the present location (and her destination) on some cognitive map; or perhaps more simply he has to know how himself to get from where she is to where she wants to go. So this de re claimis grounded in a plan and finally in a capacity for actin. It's not knowledge that that's indicative or even constitutive of de re attitude claims; it's knowing how to realize a cerain goal. Boer and Lycan (1986) propose that de re knowledge be understood relative to purposes. I take their proposal to be basically correct. But they still analyze de re attitudes in light of knowledge that - viz. knowledge of a proposition containing an attributive description. and while this is sometimes the case, it need not be; in the map task it may be the ability to point to a loction or to put an agent in a particular location that constitutes knowledge de re of that location. Boer and Lycan are interested in stopping the "regress" of "who is X?" type questions. But in so doing they conflate the issue of de re knowledge claims with their justification. De re knowledge is just a matter of having access to the object that is sufficient for accomplishing the contextually given goals at hand. The upshot of our proposal for contextual anchoring amounts to the following view of de re attitude: there isn't any absolute de re knowledge; there's de re knowledge relative to various goals that one might have.

\subsection{Previous approaches to Contextual Anchoring}

The description given of the phenomenon of contextual anchoring of the presupppositions of definites is a quite different picture of deictically used definites than that found in the philosophical and linguistic literature. Here are some approaches that might be useful to combine with the Boer and Lycan analysis of de re attitudes I have sketched above.

- contextual evaluations for indexicals and demonstratives (Kaplan 1978)

- value loading (Barwise and Perry 1983)

- DRT's external and internal anchors (e.g., Asher 1986)

Each one of these theories gives us an account of the satisfaction of the definites. Both Kaplan and Barwise and Perry suggest that a definite may be evaluated in the present context or for Barwise and Perry in any "conversationally salient" situation. This "value loading", or "externalist" type of evaluation yields a singular proposition for the sentence containing the definite. Such an account yields a connection between deictically used definites and de re attitudes, which seems needed to account for the rhetorical function of such definites. Once an agent accepts such a singular proposition or comes to believe it, he has a de re believe.

The problem is that this act of acceptance and the de re attitude as an attitude toward a singular proposition doesn't by itself link up easily to the conversational patterns we've already discussed. Consider again the position of Phillippe in (8f.i). Suppose that he accepts Isabelle's assertion whose interpretation yields a singular proposition. By accepting this assertion, he comes to have a belief, in this case a de re belief. But on the other hand, we'd like to distinguish this case from the sort of attitude that Phillippe requires in order to satisfy his conversational goals. For instance, in (8i), it's clear from his response that Phillippe doesn't have access to that contextual evalua- 
tion, which is something that these approaches can't explain. And this lack of access drives his response to Isabelle's assertion, and in particular her use of the definite $a u$ bout de l'avenue Jean Jaurès.

An alternative, "internalist" approach to singular propositions and the attendant construal of de re attitudes is to look for some internal, cognitive aspect of these attitudes. This is also a familiar idea in philosophy, also made famous by Kaplan though this time it's Kaplan's (1968) paper 'Quantifying in'. Kaplan's idea was that a de re attitude involves a particular sort of name, a "vivid name", for the object and that name as a constituent of the attitude object. Vivid names for a particular attitude holder are ones that have a lot of information associated with them, perhaps information suficient to identify the object. But, at least on this construal, vividness isn't necessary for some de re knowledge claims. Knowledge who, for instance, is presumbaly a kind of de re attitude. So now consider the de re knolwedge involved in knowing who lost the battle of Hastings for the purposes of a history exam. Here the name of a long dead Anglo Saxon king will suffice; what seems important in this case is not the amount of information as the disposal of the possessor of the attitude but the way that information interacts with the conversational goals at hand. The practical activity in the example about King Harold is just being able to supply the correct answer. We could reconstrue vividness in terms of knowing how, but we would still need to supplement this with an account of how this attitude toward the referent of the definite interacts with discourse. And we lack here any connection with accounts of presupposition.

A DRT approach to deictically used definites could make use both of internalist and externalist components. In the terminology of Asher (1986) (see also Kamp 1987), a DRT approach to deictically used definites could make use both of external anchors to simulate the truth conditional effects of singular propositions and of internal anchors that could furnish additional descriptive conditions. The definite would introduce a discourse referent $x$ that would be linked to some object $a$ in the context via an external anchor, which would ensure that the satisfaction of the discourse representation must proceed by assigning a to $\mathrm{x}$. Additionally, the binding of the presupposition generated by the definite could take place via an internal anchoring of $\mathrm{x}$ to some discourse referent in a representation that is part of the agent's cognitive state. Such internal anchors link the interpretation of one discourse referent $\mathrm{x}$ in one representation $R_{/}$to the interpretation of another discourse referent y in another representation $R_{2}$; more precisely, we say that a pair of assignments $\mathrm{f}, \mathrm{g}$ satisfies $R_{/}$and $R_{2}$ respectively given an internal anchor between $x$ and $y$ iff $f(x)=g(y)$. Unlike the value loading accounts, this approach focuses on the cognitive aspect of these contextual anchorings.

DRT approaches also give us an anaphoric account of presuppositions. Roughly, a definite description introduces a presuppositional component into the discourse representation in which a discourse referent is introduced along with the properties that are given by the description. This discourse referent must be linked to some other discourse referent in the context, unless the presupposition is to be accommodated. We can now postulate that the discourse referent introduced by presupposition generated by the deictically used definite binds via an internal anchor to some discourse referent in the interpreter's cognitive state. Nevertheless, a DRT approach says little about the sort of knowing how that we've seen is important in the examples. The uses of definites in these dialogues establish that it's the cognitive access for certain purposes that are crucial for de re attitude claim. Like Kaplan's own picture of de re knowledge in quantifying in, DRT's conception of internal anchoring lacks any tie to practical plans; in fact there aren't any constraints on internal anchors whatsover, which might well 
accord with our intuitions about beliefs of a certain kind (footnote Jeshion here) but which doesn't capture the particular sense of de re attitude at issue here. The proposal I've just developed as it stands is still just binding, albeit to a belief context rather than a discourse context. We need some story here of familiarity that goes beyond binding. We need an account in which, e.g., the variable associated with Isabelle's location is linked to some cognitively accessible discourse referent in a way that allows Phillippe to accomplish his conversational goals.

Let's see how this might be cashed out in terms of the examples in the dialogues. The thesis about de re knowledge claims goes hand in hand with a goal relative notion of contextual anchoring. Fleshing out this idea is what I turn to now. I'll elaborate a theory of presupposition and of the logical form of presupposition triggers (though not too much hangs on this) which allows a wide variety of presupposition bindings. I'll also say something about how this account interacts with a theory of cognitive modelling. I'll then return to these contextual anchorings.

\section{SDRT's account of presupposition}

I turn now to see how to analyze anchoring uses of definites within the anaphoric account of presuppositions of Asher and Lascarides (1998a, 1998b). I nee, however, to give a few more details of the account than I did earlier. In this account presuppositions are, like assertions, units of information that must be integrated into the discourse context. A unit of information, however, can be integrated into the discourse context in different ways, ways which correspond to the rhetorical function of that unit of information. Accordingly this leads us to a more complex notion of a discourse structure than that present say in DRT, A discourse structure is a pair $(A, F)$, where:

- $A$ is a set of labels

- $\Phi$ is a set of formulas representing clauses and relations on labels (between clauses)

- $F: A \rightarrow \Phi$

We'll express the effects of $\mathrm{F}$ on A via the notation $\pi: K$.

While both assertions and presuppositions must be integrated to the discourse context, presuppositions must be linked via particular discourse relations. Asher and Lascarides (1998b) isolated two, Background and Defeasible Consequence. While there is no accommodation per se in this framework since the attachment of presuppositions is just part and parcel of building a discourse structure, the cases of accommodation in the literature correspond to linking the presupposition via the relation of Background. Defeasible Consequence generalizes the propositional binding relation in van der Sandt and Geurts, while Background imposes thematic constraints that the notion of accommodation lacks. Background $(p, q)$ holds iff $q$ and $p$ entail a common topic and $q$ specifies properties of elements in $p$ that set the stage for or serve as an explanation for some event described in $p$ or in some proposition linked to $p(\neq q)$.

As discussed in the introduction, presuppositions are understood as containing incomplete or underspecified elements. This is particularly true in the case of the presuppositions of definites. In discussing earlier examples, I alluded to an innovation of the SDRT view that incorporates an underspecified bridging relation $B$ in the presup- 
position of a definite. The representations of presuppositions underspecify the discourse relation by which they attach to the discourse context and the other term of that relation; as such they are explicitly anaphoric elements whose proper interpretation must resolve all of these underspecifications - i.e. find appropriate specifications for these underspecifications.

Here's an example of how a presupposition and an assertion would be analyzed in SDRT. The asserted component is labelled with $a$, the presupposed part with $p$. The asserted part produces a labelled SDRS for for the asserted content of a clause; another SDRS - viz.(11b), the " $\mathrm{p}$ " part - with speech act discourse referent $\pi$ ', and condition $\pi$ ' : $K_{\pi^{\prime}}$ for the presupposed content of this clause, where $K_{\pi^{\prime}}$ will be the DRS discourse constituent that represents the presupposition.

The man walked.

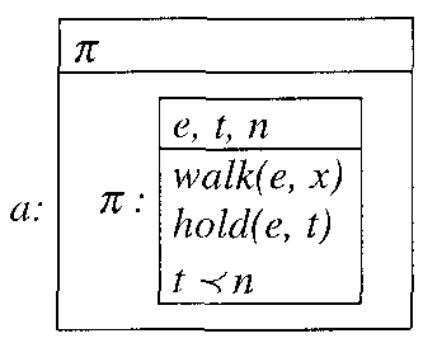

$$
\begin{array}{|l|l|}
\hline \pi^{\prime}, R, v \\
\hline \pi^{\prime}: & \begin{array}{l}
x, u, e^{\prime}, t^{\prime}, B \\
\text { man }(x) \\
B\left(e^{\prime}, x, u\right) \\
h o l d\left(e^{\prime}, t^{\prime}\right), \\
B=? \\
u=?
\end{array} \\
R\left(v, \pi^{\prime}\right) \\
R=? \\
v=?
\end{array}
$$

In the SDRS above, the man denoted by the definite must be (bridging) related to an antecedent object (so (11) couldn't be uttered in a null context). Further, the presupposition must be bound to the context via a rhetorical relation. While both presuppositions and assertions must get integrated into the context, they do so in different ways. Presuppositions link typically with either Defeasible Consequence or Background. We'll add here the relation of Anchoring as another relation that presuppositions can bear to other elements in the discourse structure. When the components above are attached to the discourse context and the various specifications of underspecified conditions are effected (as far as possible), then we have an update of the discourse context with the information given by (11). Following Asher and Lascarides (1998b), I'll represent update by a three place relation involving the discourse context, the new information and a "resulting" SDRS that integrates the new information into the discourse context.

In the introduction, I also mentioned that SDRT incorporates a principle of resolving $B$ to identity whenever feasible, thus capturing the preference for binding that is a feature anaphoric theories of presupposition. This accounts for the simple cases of binding in

Whenever I see a book in a bookstore that I like, I try to buy the book.

We can formalize this principle as follows: 
- If Possible Use Identity:

$$
\left(K_{\beta}\left[B=\text { ?] } \wedge \downarrow \operatorname{Update}\left(K_{\tau}, K_{\infty}, K_{\beta}[B / \lambda x \lambda y x=y]\right)\right) \rightarrow\langle\tau, \alpha, \beta[B / \lambda x \lambda y x=y]\rangle\right.
$$

This constraint says that as long as setting the bridging relation to identity is well defined $\downarrow$, then the update of the discourse context with $\mathrm{K}$ fi will set B to identity.

As we saw in (1) or (2), sometimes we cannot resolve B to identity. In that case whathappens? When we try to resolve the bridging relation to something other than identity, we do so in a way that maximizes dis-course coherence. Since the update relation is nondeterministic, there are often many ways new information can be integrated into a discourse con- text. Sometimes the resolution of underspecified elements as in (1) will determine how the new information attaches to the discourse context. Some of these ways provide for a more coherent discourse than not. Attachment and resolution of underspecified elements always tries to maximize discourse coherence. And to give this constraint some bite, I specify some things about the preference order $\succ$ on discourse structures:

- More specified, well typed SDRSs are always preferred to SDRSs with less specification $-\tau \rightarrow * \tau^{\prime} \rightarrow \tau^{\prime} \succ \tau$.

- SDRSs that violate type restrictions are less preferred than those that don't violate such restrictions.

- defeasible consequence $\succ$ background for presupposed material.

- background with a more specific topic $\succ$ background with a less specific topic.

- where speech act related goals or SARGs can be inferred from Cognitive Modeling, a discourse structure that is more likely to lead to SARG satisfaction is more coherent than one that is not likely to lead to SARG satisfaction.

All of these constraints on $\succ$ require probably more explanation than I can give here. The first constraint just says that if an SDRS with fewer underspecifications where no type constraints on predicates are violated is to be preferred to an underspecified SDRS. The second constraint says that anytime a type restriction is violated that SDRS is less preferred to other SDRSs where the type restriction is not violated. The third constraint says that some discourse relations between presupposed material and the discourse context like defeasible consequence are to be preferred over a relation of background between the presupposed material and the discourse context (thus encoding an anaphoric theory of presupposition's preference for binding over accommodation). The fourth constraint tells us that the tighter the connection between the background material and the foreground material, the better the discourse coherence between those two segments, as a tighter connection between background and foreground will allow for a narrower, or more specific, topic. Thus, in an example like (1) maximizing discourse coherence or MDC will prefer those SDRSs where the bridging relation in the presupposition of the definite is set to some relation other than identity since setting the relation to identity would require the identification of a couple with a woman, which violates type restrictions. But further setting the bridging relation to be "a member of" is preferred on several counts: it specifies the underspecified relation and it also gives rise to a Background relation with a more specific topic than would be otherwise possible. For the inferential binding cases like (4), MDC will specify the bridging relation to the 
witness relation as we described earlier, because that will allow us to attach the presupposition with the relation of defeasible consequence to the SDRS consisting of the first two sentences of (4), and that is preferred to any option on which the presupposition is not so attached. Below I give a picture of how the specifications would go. Def-cons is the relation of Defeasible Consequence, Commentary is another relation in which the speaker of the second constituent expresses an attitude toward some element in the first constituent.

\begin{tabular}{|c|c|c|c|}
\hline \multicolumn{4}{|c|}{$\pi, \pi_{p}, \pi_{c}$} \\
\hline \multicolumn{4}{|c|}{$\operatorname{Def}-\operatorname{cons}\left(\pi, \pi_{p}\right)$} \\
\hline Con & mentary $\left(\pi, \pi_{i}\right)$ & \multirow[b]{3}{*}{$\pi_{p}$} & $x, u, e^{\prime}, t^{\prime}, B$ \\
\hline & $\pi_{c}, \pi_{b}$ & & donkey $(x)$ \\
\hline$\pi:$ & $\begin{array}{l}\text { Instance }\left(\pi_{a}, \pi_{b}\right) \\
\pi_{a}: K_{4 a} \\
\pi_{b}: K_{4 b}\end{array}$ & & $\begin{array}{l}B(x, u) B=W \\
u=\llbracket \text { a donkey } \rrbracket \\
o w n s(x, j)\end{array}$ \\
\hline \multicolumn{4}{|c|}{$\pi_{c}: K_{4 c}$} \\
\hline
\end{tabular}

That leaves our last constraint on $\succ$ for discussion It has to do with cognitive modelling, the part of our story that I turn to next.

\subsection{Cognitive Modeling}

As we've seen anchoring requires linking an epistemic attitude to conversational goals. Thus, we need to be able to infer conversational goals from conversational patterns. In other work (Asher and Lascarides 1998, Asher 1999), Lascarides and I have co opted some of the insights of Gricean pragmatics and speech act theory to link speech act related goals or SARGs to discourse structure. On our view, the rhetorical relations in dialogue bring considerations about why participants ask, elaborate, request, assert and respond to what is said. In turn such SARGs help elucidate and further constrain discourse structure. In order to formulate a precise notion of anchoring for deictically used definites, I will give some of the principles for discovering SARGs in that component of SDRT that supplies a rough cognitive model of discourse participants.

A second feature of anchoring is that once the anchoring function of a deictically used definite is accepted by the interpreter, it appears that speaker and hearer mutually believe that the definite picks out the same object. Given that we have adopted a largely internalist view of the de re attitude involved in anchoring and that the way dynamic semantics models attitudes has nothing to say about knowing how, I'll show how such mutual belief can be derived from axioms having to do with the beliefs of the participants.

Cognitive modelling in SDRT follows the basic BDI approach in which we have modal operators for belief (K45) $(B)$ and intention $(I)(\mathrm{KD}) \mathrm{s}$, and a mutual belief operator $M B_{G}$, for any group $G$ with the usual axiomatization.We'll assume distributivity of $B$ and $I$ over $>$, as well as the $\mathrm{K}$ axiom. I'll suppose that $B_{A} p_{\pi}$, corresponds to $\mathrm{A}$ believing the proposition content represented in the SDRS $K_{\pi}$. It is assumed in SDRT that whenever an agent intends something, he does not already believe that it is true: 
$I_{A \varphi} \rightarrow \neg B_{A \varphi}$. Goals are propositions that one intends (a simplification but good enough for our purposes here). I'll start with the simple Grice like axioms for belief modelling. The first axiom allows us to infer beliefs from assertions.

- Sincerity: $R(\alpha, \beta)>\operatorname{Bel}_{\mathrm{Agent}(\beta)} R(\alpha, \beta)$

A second default, competence, transfers the beliefs of one agent to another, while the constraint on acceptance gets us from acceptances to beliefs about what others have said.

- Competence: $B_{A} \Phi>B_{B} \Phi$

- Constraint on Acceptance: $\operatorname{Accepts}(\alpha, \beta)>M B_{\text {Agent }(\beta)} \alpha$

Let's now turn to the inference of SARGs. Inferences concerning SARGs also revolve around a Gricean notion of cooperativity. One agent B is cooperative with another agent A if he adopts A's goals. According to this, B will try to realize A's goals in so doing help A. This can be only a default, because there may be many times when B has conflicting goals with respect to A. So, a second level to cooperativity is to indicate if the speaker does not share the conversational goals of the other participant. These principles are expressed by the following axiom:

\section{- Cooperativity:}

(a) $I_{\Lambda}(\Phi)>I_{B}(\Phi)$

(b) $I_{\Lambda}(\Phi) \wedge \neg I_{B}(\Phi 0)>I_{B} B_{A} \neg I_{B}(\Phi)$

Cooperativity doesn't tell us what an agent's goals might be in dialogue, because it may not be possible to infer an agent's goals from what he says. This is where particular linguistic axioms like QRG and RRG come in.

- Question Related Goals (QRG):

$\operatorname{QAP}(\alpha, \beta)>\left(I_{\text {agent }(\alpha)} B_{\text {agent( } \alpha)} \beta\right)$

This axiom states: if $\beta$ is the answer to the question $\alpha$, then normally the agent or speaker of $\mathrm{ff}$ intends to be in a certain state in which $\beta$ is true. This axiom applies whenever an agent asks a question. A similar axiom holds for requests.

- Request Related Goals (RRG):

$$
\alpha: !>I_{\text {Agent }(\alpha)} a
$$

SARGs for assertions are more difficult to capture. We'll assume that knowledge relevant to connecting the content of assertions, which we'll assume here to be sincere, to their conventionally associated SARGs can be accessed by the linguistic system. Finally, we'll assume that if we compute a SARG via Cooperativity or RRG or QRG, then if the agent's speech act has both a presuppositional and an assertional component, the SARG computed applies to both.

One final matter is that in SDRT questions can elaborate on other questions or requests. We see this in ( $8 \mathrm{~b})$ already where Phillippe's question is intended to help 
elaborate a plan already implicit in Isabelle's opening (8a). We call the rhetorical relation that (8b) stands in to (8a) Question Elaboration or QElab (see Asher 1999, Asher and Lascarides 1998c). If we have a Qelab, then the SARGs of the second question include the SARGs of the first. Formally, we express this as:

- SARG additivity:

$(\mathrm{Q}-\operatorname{elab}(\alpha, \beta) \wedge \operatorname{SARG}(\alpha, \delta)) \rightarrow \operatorname{SARG}(\beta, \delta)$

This ensures that Qelab SARGs are carried along as discourse participants try to answer the original question by asking other questions. We'll group the SARGs that are accumulated through nested Qelabs within a cluster.

\section{Conversational Goals and $D e$ Re Attitudes}

We have most of the parts in place for our presuppositional account of contextual anchoring. We needed an account of conversational goals, and we have just seen ways of getting SARGs from various conversational moves. We determined earlier from looking at our examples that these conversational goals were essential in determining the de re attitudes that are part of contextual anchoring. Further, I argued that these de re attitudes were really grounded not in an attitude toward a proposition but in an ability. I'll try to be more precise about what this ability consists in now using the devices available to a theory like SDRT. That means giving some analysis of this practical capacity in terms of a broadly DRtheoretic account of belief.

Let's return first to the turn (8ef). Isabelle first corrects her previous turn and then tries to answer Phillippe's question in (8b). Isabelle uses a deictically used definite, 'the end of the avenue Jean Jaurès' in her answer, that Isabelle further localizes with reference to 'the big square' (la grande place). What is the discourse relation between the presupposition of the definite and the discourse context? Presumably, the presupposition is to anchor the assertion. Interestingly, Isabelle goes on to elaborate on this location where she is, and the point of this elaboration in (8e.ii) is ostensibly to help establish the Anchoring relation between the presupposition of the definite and the discourse context.

To appreciate the cognitive effects of Anchoring, let's see what happens if the discourse move by Isabelle which includes the anchoring is accepted by Phillippe. If this Anchoring relation is accepted by Phillippe, it has a certain implication: that Phillippe will be able to determine which location Isabelle describes. Moreover, Phillippe's knowing where Isabelle is is the SARG derived from (8b) via QRG. By SARG additivity this remains a SARG through (8d). ${ }^{3}$ By Cooperativity Isabelle takes over this SARG and she is trying to satisfy that SARG with her utterance of (8e). Were (8e) to be accepted, she would have satisfied that SARG and perhaps also the associated SARG of getting her unlost. In (8f), however, Phillippe doesn't accept the Anchoring relation, which is why he asks "Wait a minute, I don't quite see where you are."

So accepting an Anchoring relation between the presupposition introduced by a definite $\psi$ and some element in the discourse context by an agent $\mathrm{A}$ requires a

Actually, in SDRT theoretic terms, (8d) attaches to (8b) via Question Elaboration or Q-elab, which automatically propagates the SARG of the first question forward, but I'll gloss over the details of this part of the discourse structure here. 
computable means of getting to the referent of $\psi$ from the present here and now, the present nonlinguistic context of utterance, for some given purpose $\varphi$. To this end, I define a Path relation on discourse referents $x$, which is introduced by the presuppositional component of the definite's DRtheoretic lexical entry and $u_{1}, \ldots, u_{\mathrm{n}}$ relative to a SARG $\varphi$ and its associated cluster, $\mathrm{P}_{\varphi}\left(\mathrm{A}, x, u_{1}, \ldots, u_{\mathrm{n}}\right)$. This relation holds iff

- $u_{1}, \ldots, u_{\mathrm{n}}$ are accessible in A's belief state and some of the $u_{1}, \ldots, u_{n}$ are externally anchored to distinguishable objects in the present context (e.g., the here and now).

- there is a collection of formulas $\mathrm{T}\left(u_{1}, \ldots, u_{\mathrm{n}}\right)$ characterizing correct beliefs of $\mathrm{A}$ concerning $u_{1}, \ldots, u_{\mathrm{n}}$ such that $\mathrm{A}$ has a proof from $\mathrm{T}\left(u_{1}, \ldots, u_{\mathrm{n}}\right)$ that $\varphi$.

Thus Anchoring as a discourse relation between a presupposition introduced by a definite and some other element in the discourse context in the SDRS for an agent A entails that the Agent can satisfy a current SARG that he has. The connection to a particular de re attitude grounded in an ability comes about because in many, and perhaps in all cases, the SARG that needs to be satisfied specifies a de re attitude (as in our dialogue examples) or requires for its satisfaction a de re attitude that is itself grounded in an ability. That is, satisfying a SARG may often involve a practical ability in addition to beliefs towards attitudes.

Spelling out the entailment without specifying the SARG further seems difficult. On the other hand, in the case of knowledge where, which is what is at issue in the examples culled from the dialogues (8) and (10), we can be more specific. In such cases the path formula could plausibly involve a sequence of locations $l_{l} \ldots l_{n}$, such that $T$ and $l_{l}$ have the following features:

- formulas of the form $C\left(l_{i}, l_{i+1}\right)$ for $1 \leq \mathrm{i} \leq \mathrm{n}$, where $C$ is the relation of Connectedness.

- $T$ determines distance and orientation information for each $l_{i}$ and with respect to $l_{i-1}$ and $l_{i+1}$ and

- the initial location $l_{l}$ is an accissible point in the present non-linguist context.

The idea is that if the dialogue agent whose SDRS contains an Anchoring relation and the associated goal is knowing where someone is, then the agent should be in possession of information that will allow him to compute a path, a sequence of connected locations that will get him from his present surroundings to the location denoted by the definite. Or if the SARG is a slightly more complex type of knowing where - say the goal is to know where something a is relative to some other location 1 , then the agent must be in possession of a path from the location of a to $l$.

With this in mind, let's once again go back to the exchange in (8ef). Phillippe doesn't accept the Anchoring relation. Why? Well, it's manifestly because even though Phillippe presumably knows what the end of ave. Jean Jaurès is, he doesn't know where she is. In this case the demands of his SARG to know where she is, can only be satisfied if he can bind the definite to a spatially determinable object from the present context i.e. have his belief structure satisfy a path condition between where Isabelle is and his current context, or perhaps where she needs to go. As his response makes evident, he cannot. 
Other examples from our dialogues bear out the usefulness of thinking in terms of $\mathrm{T}$ as determining a path. In (10) speaker A uses definites that he can link to elements in hisenvironment and intends to have B link to elements in his immediate context (the map). A has presumably already linked the discourse referents via a Path condition. B stops the flow of instructions when he cannot determine a Path relation. The Path condition is really a constraint on a dialogue agent's attitudes. Path binding is a type of internal anchor in DRT. But what is distinctive about it is its link to practical activities as defined by the discourse and by SARGs.

\section{Contextual Anchoring as Discourse Function}

We have now seen how Anchoring as a discourse function has entailments concerning de re abilities. What remains to be done is to specify how we might infer Anchoring as a discourse relation and to specify formally the relationship between the discourse structure and the cognitive constraints. This will be done through a pair of axioms written in the SDRT format.

Like other discourse relations, Anchoring can also specify the underspecified bridging relation in the presupposed information. Given our informal analysis, we might think that Anchoring should somehow specify the bridging relation to a path relation. Here as with inferential binding, there is a downward flow from the global discourse structure and its associated cognitive model to resolving certain underspecifications needed in the binding of presuppositions.

In those examples of inferential binding, however, the bridging relation cannot be set to identity without violating type restrictions. Here the situation appears to be different. Consider the exchange in (8gh). Isabelle tentatively accepts $(8 \mathrm{~g})$. She uses a Path condition to bind the railroad tracks to something in her immediate surroundings. The path sequence has length 1 , and she has information about the direction and distance that makes it more likely that her current SARGs will be satisfied: the SARGs are that Phillippe know whether Isabelle has passed under the railroad tracks (inferred via QRG as a SARG for Phillippe and then as a SARG for Isabelle via Cooperativity), that Phillippe know where Isabelle is and that Isabelle find her way (inferred via QRG, Cooperativity, SARG additivity). But she's not sure, so she tells Phillippe what the head of the Path sequence is in (8h).

Now how does the Path condition interact with the specification of the bridging relation? Given the instructions given earlier by Phillippe to Isabelle, it's easy enough for Isabelle to set the bridging relation to identity. This would be sufficient to bind the presupposition via Background to the asserted constituent or to Background's topic. But this won't achieve Phillippe's SARG, which is determined by his question - namely, this is the SARG of knowing whether Isabelle passed under the railroad tracks he told her about. Further, we can assume that Isabelle also has the SARGthat Phillippe know whether she went under the railroad tracks or not. This follows from QRG and Cooperativity: QRG tells us that Phillippe has as a SARGthat he know the answer to his question; Cooperativity transfers this SARG from Phillippe to Isabelle.

In order to satisfy this common SARG, Isabelle has to do two things; she does indeed have to link the railroad tracks mentioned to those given in Phillippe's instructions, and she has to bind the location of that bridge to some location in her journey or where she is now. And if this analysis is right, then we need both to have Anchoring determine a Path condition while also allowing in the relevant cases the 
bridging relation to be set to identity. This would result in the most coherent discourse structure according to MDC because it leads to a satisfaction of a given SARG and it is also the one mandated by If Possible Use Identity. So it appears that whenever setting the bridging relation to a Path relation would help achieve some recognizable SARG, we infer Anchoring as a discourse relation; and in turn Anchoring then determines the existence of a Path condition relation. But an inference to an Anchoring relation doesn't clash with the principle of setting the bridging relation to identity If Possible Use Identity; rather it complements it.

I have formalized this using the underlying nonmonotonic logic of SDRT. We infer Anchoring by default whenever resolving B to a path relation would normally allow the agents involved to see to it that (formalized via the operator stit) their SARGs are realized. Below we use $[B=?](\beta)$ to mean that $K_{\beta}$ has the underspecified conditions $\mathrm{B}=$ ?.

- Anchoring:

$\left\{\langle\tau, \alpha, \beta\rangle \wedge \operatorname{Sarg}(\beta, \phi) \wedge[\mathrm{B}=?](\beta) \wedge\left(K_{\beta}\left[B \rightarrow * P_{\phi}\right] \rightarrow \diamond \operatorname{stit}(\operatorname{agent}(\beta), \phi)\right)\right\}>$ $\operatorname{Anchor}(\alpha, \beta)$

- Constraint on Anchoring:

(Anchor $(\alpha, \beta) \wedge[B(x, y)](\beta) \wedge \operatorname{Sarg}(\alpha, \beta)) \rightarrow$

$\left(K_{\alpha} \wedge K_{\beta} \wedge\left(B_{A} \operatorname{Anchor}(\alpha, \beta) \rightarrow\left[v^{\rightarrow} \mid \operatorname{Path}_{\phi}\left(a, x, v^{\rightarrow}\right)\right]\right)\right)$

The constraint on anchoring says that an anchoring relation entails that its terms must be true propositions and further that if an agent believes $\operatorname{Anchor}(\alpha ; \beta)$, then the Path condition must be satisfied by agent A. Let's now see how this axiom works. Let's go back to $(8 \mathrm{~g})$ and its context once again. Isabelle first processes Phillippe's question. She isolates out the presupposition of the definite la voie ferrée in a constituent $\mathrm{K}_{e, i i, p}$ and the assertion $\mathrm{K}_{e, i i}$. Given what we have said earlier about cooperativity, she attemptsto cooperate with Phillippe's SARG of knowing the answer to this question and she does her best to tell him. But in order to give him an answer, she has to be able to anchor the presupposition and thus satisfy a Path condition linking the bridge to some element in her trajectory, which I assume can be reconstructed from her here and now, or in her here and now itself. So Isabelle's SDRS looks something like this, if we ignore the processing of definites like Phillippe and you and the manner adverbial clause:

(8e.ii)

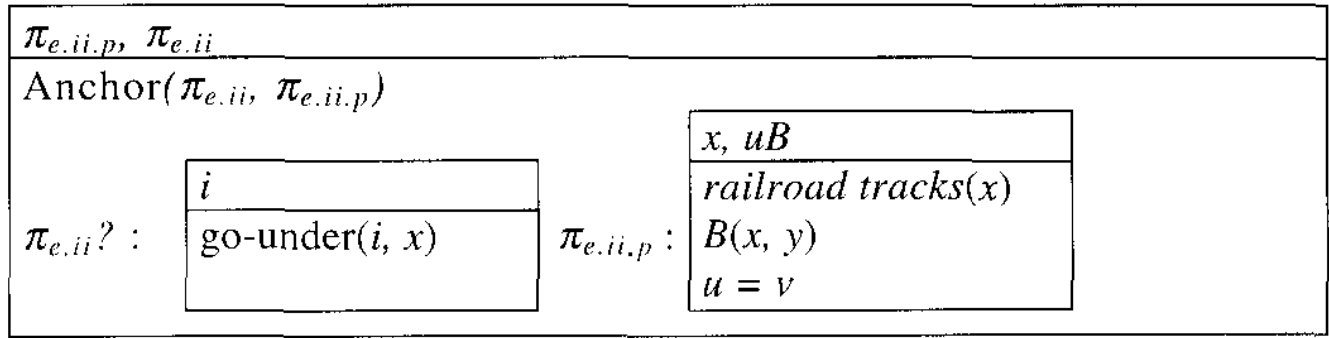

In the above, $v$ is the discourse referent for the railroad tracks introduced in Phillippe's previous instructions and one that is presumably now cognitively accessible in Isabelle's cognitive state The bridging relation has been set to identity between $\mathrm{x}$ and $\mathrm{v}$. $\mathrm{u}$ is some discourse referent in Isabelle's cognitive state that is an internally anchor for the presupposed material. And it is in virtue of $u$ that the Path condition is satisfied. 
But Isabelle is not sure whether the Path condition has been satisfied. So she attempts to clarify or elaborate on what the putative Path condition is that she has found for $\mathrm{x}$. In SDRT we model this rhetorical function by attaching (8g) to $\pi_{\text {e.ii.p }}$ with the discourse relation Elaboration (for details on this relation see Asher 1993, Lascarides and Asher 1993). This Elaboration also constitutes an indirect answer (Asher and Lascarides 1998a) to the question in $\beta$ e.ii , and it is precisely this Elaboration and indirect answer that Phillippe rejects in $(8 \mathrm{~h})$.

Let's see how our approach fares with discourse initial deictic definites. Consider the initial turn (10a) where A mentions the tribal settlement. Here there is presumably no discourse referent already introduced in the discourse that could serve as a link, and so resolving the bridging relation to identity in this case is not possible. We'll assume that $\mathrm{B}$ is able to anchor the presupposition generated by the extinct volcano, but as his question demonstrates, he is not able presumably to determine a Path condition for the discourse referent $\mathrm{x}$ introduced by the tribal settlement. So presumably the question in (10b) is intended to help get an appropriate Path condition for $\mathrm{x}$ and once that question is answered B can anchor the presupposition to the asserted content of (10a). In this case since 'If Possible Use Identity' because this axiom cannot be used, MDC resolves the Bridging relation to the Path condition, once the Anchor relation is established.

\section{From Acknowledging Path Binding to Mutual Belief}

A final element in the analysis of anchoring is to account for the fact that when an Anchoring function has been acknowledged, the two participants in the conversation have the mutual belief that the Path relations link to the same location. This comes about after the interpreter accepts an Anchoring relation proferred by the speaker; this means that the Path condition is satisfied not only by the speaker but the interpreter as well. Because the Path relation must be satisfied by both the speaker's and the hearer's beliefs if Anchoring, we say that Anchoring is a kind of coordination.

How do we acquire mutual belief in communication? Due to Fisher (1988) we know that if communication is synchronous, then mutual belief can be had. Suppose there is enough simultanous exchange of information to have it qualify as synchronous. This is in fact encoded in our constraint on agreement: a signal of agreement to a previous contribution in which a discourse structure like Anchoring holds gets us to a mutual belief that the presupposed material is serving as an Anchor. Now consider any of the conversational turns where an Anchoring relation is proposed and then accepted (e.g., 10cd).

(10) c. A: It's at the bottom. It's to the left of the a extinct volcano.

d. B: Right. How far?

By sincerity we have that $A$ attaches the presupposed material given by the extinct volcano with Anchoring to his turn. By signaling an agreement with Right $B$ also adopts this discourse structure for A's turn. We can now conclude given our assumptions that there is mutual belief in this discourse structure (and that if you will we have that discourse structure in the common ground). But now how to we get to that mutual belief that both path bindings link to the same object? How do we even represent this fact? We can relatively easily answer the latter question: among the beliefs of a dialogue agent $A$ are also beliefs about other dialogue participants-let's say for the 
moment just $B$. Given that there is a shared belief that both participants have a path binding (from Anchoring), $A$ can internally anchor the last discourse referent $u$ of B's Path condition as in Asher (1986) or more recent work of Kamp. Here I'm going to use the older notation and represent internal anchors as equalities within the embedded belief context. So we'll represent this internal anchoring for $A$ as an equality $x_{A}=$ $x_{B}$ in A's representation of $B$ 's belief state and similarly for $B$. In effect this says that B's beliefs about $x_{B}$ are also in effect a belief about $A$ 's individual concept.

We'll suppose that Anchoring has been proposed and accepted as in (10cd). So it's mutually believed that each agent's cognitive state satisfies the Path condition for the discourse referent introduced by the presupposition of the definite. This means:

- Assume $A$ represents $B$ 's cognitive state as having a formula $\phi\left(x_{B}\right)$ in it for the definite while his own has $\phi^{\prime}\left(x_{A}\right)$.

- $B_{A} B_{B} \phi\left(x_{B}\right) \wedge B_{A} \phi\left(x_{A}\right)$.

- By competence we have $B_{A}\left(B_{B} \phi\left(x_{B}\right)>B_{A} \phi\left(x_{B}\right)\right.$.

- By distributivity of belief over > and DMP: $B_{\Lambda} B_{B} \phi\left(x_{B}\right)$, which then in K45 yields

- $B_{A} \phi\left(x_{B}\right)$, and so by $\mathrm{K}$

- $B_{A}\left(\phi\left(x_{B}\right) \wedge \phi^{\prime}\left(x_{A}\right)\right)$.

And since $\phi$ entails a uniqueness clause, first order logic yields:

- $B_{A} x_{A}=x_{B}$

- Since this is derived from mutually believed information, $B$ can pursue the same reasoning and reason that $A$ has also done this reasoning. Hence by our jump to the mutual belief axiom, we get

- $M B_{A, B} x_{A}=x_{B}$

That seems to me to suffice for internal anchoring however it's represented. Notice that postulating this equality in $A$ 's belief state leads to no binding problems because $A$ supposes through competence that there is an $x_{B}$ of which $B$ has his beliefs.

\section{Conclusions}

I've shown that a discourse based, anaphoric theory of presupposition has an interesting story to tell about at least some deictic uses of definites. In many of these uses presuppositions are anaphorically bound to the discourse context via a particular discourse relation, Anchoring, whose semantics and conversational function is directly linked to the participant's conversational goals. Anchoring entails a de re attitude, but it is one that is linked to an increased capacity for satisfying at least some conversational goals. Our investigation has confirmed the view that de re attitudes involve some sort of knowing how. We have seen how Anchoring, when accepted by all participants, leads to a mutual belief in coordinated reference - viz. that all the particpants are referring to 
the same thing and can single it out at least insofar as that's required for conversational purposes. SDRT gave us the framework within which to analyze the discourse function of these uses of definites, and the modest set of defaults that SDRT uses in developing a theory of conversational goals or SARGs was helpful in deducing SARGs for the Anchoring analysis.

Further tasks: Presumably definites outside the context of spatial localization dialogues can also be Anchored. So one idea for further research is to see how to extend this analysis to other definites - deictically used pronouns and the like. Moreover, it seems that almost all words have presupposition like associated information whose failure to be anchored (bound) lead to similar corrections as those we've studied here. Consider these metalinguistic bits of anchoring information in the examples below due to Ginzburg that are called into question by B's responses.

(14) a. A: John kowtowed.

b. B: Kowtowed?

(15) a. A: Chris inebriated Pat.

b. B: Inebriated?

\section{References}

Asher, N. (1986) Belief in Discourse Representation Theory, Journal of Philosophical Logic 15, 127-189..

Asher, N. (1993) Reference to Abstract Objects in Discourse, Kluwer Academic Publishers.

Asher, N., J. Busquets and A. Le Draoulec (2001) Cooperativity in Dialogue, in edd. Bras, M and L. Vieu Semantics and Pragmatic Issues in Discourse and Dialogue, Current Research in the Semantics/Pragmatics Interface, Elsevier Publishers, 217246.

Asher, N. and A. Lascarides (1998a) Bridging, Journal of Semantics, 15.1, 83-113.

Asher, N. and A. Lascarides (1998b) The Semantics and Pragmatics of Pre-supposition, Journal of Semantics, 15, 239-299.

Asher, N. and M. Morreau. (1991) Common Sense Entailment: A Modal Theory of Nonmonotonic Reasoning, in Proceedings to the 12thInternational Joint Conference on Artificial Intelligence, Sydney Australia, August 1991.

Barwise, J. and J. Perry (1983) Situations and Attitudes, MIT Press.

Boer, S. and W. Lycan (1986) Knowing Who, MIT Press.

Fisher, J. (1988) Common Knowledge and Synchronous Communication, ed. Y. Moses, Proceedings of the Conference on Theoretical Aspects of Reasoning about Knowledge.

Geurts, B. (1995) Presupposing, PhD. dissertation, Universität Stuttgart.

Heim, I. (1982) The Semantics of Definite and Indefinite Noun Phrases, PhD thesis, University of Massachussetts, Amherst.

Heim, I. (1983) On the Projection Problem of Presuppositions, Proceedings of the West Coast Conference on Formal Linguistics, 2, 114-126.

Kaplan, D. (1968) Quantifying In, Synthese 19, 178-214.

Kaplan, D. (1978) Dthat, Syntax and Semantics 9, ed. P. Cole, 221-253.

Lascarides, A. and N. Asher (1993) Temporal Interpretation, Discourse Relations and Commonsense Entailment, in Linguistics and Philosophy, 16, 437-493.

van der Sandt, R. (1992) Presupposition Projection as Anaphora Resolution, Journal of Semantics, 19(4). 